\title{
The role of the specialist physiotherapist in ambulatory emergency care; leading on developing the frailty pathway in the ambulatory assessment unit at the John Radcliffe Hospital, Oxford
}

\author{
Authors: Beverley Greensitt, Jordan Bowen, Sudhir Singh, Leila Vaziri, Anuja Bambarvajane, James Price and \\ Mridula Rajwani
}

\section{Introduction}

\section{Background}

The Future Hospital Commission report recommended ambulatory care by default for all acute presentations. The ambulatory assessment unit (AAU) at the John Radcliffe Hospital aims to provide excellent care for complex patients with varying range of medical presentations. It sees over a $30 \%$ of the acute take in a 24 -hour period ( $50 \%$ in operational hours), with over $40 \%$ of AAU patients over the age of 70 . Staff feedback consistently identified a suboptimal service provided to the frail group within this patient cohort. A dedicated physiotherapy post was appointed in October 2018 to address this.

\section{Aim}

$>$ Early identification patients with frailty on the ambulatory unit.

$>$ Improve staff understanding of frailty, assessment and referral.

$>$ Support plan in the community for patients with follow-up.

$>$ Influencing admission rate in this group of patients.

\section{Materials and methods}

> Frailty staff questionnaire to ascertain baseline understanding and learning needs.

> Introduction of frailty identification tool as per trust guidance.

$>$ Raise staff and patient awareness of available services within the community to support patients.

> Data collection to review interventions taken, bed days saved and re-admission rates.

\section{Results}

In a 2-month period following introduction of the service, 76 new patients were seen; $89 \%$ were discharged on the same day; $17 \%$ had admission avoidance directly due to therapy intervention;
$28 \%$ and $14.5 \%$ of referrals were referred to community therapy and nursing, respectively and $66 \%$ of patients were provided with information on service available. The re-admission rates within 7 days and 30 days were $3 \%$ and $1.3 \%$, respectively. Staff surveys have felt that the introduction of the service has improved the care to this group of patients. Over this short period, the number of bed days saved were 26 with a significant cost saving overall.

\section{Conclusion}

The introduction of a dedicated therapy service to an ambulatory setting has shown to have a role in influencing admission and the care provided to frail patients. There is still room for further education and potentially increasing capacity of the service by expanding the workforce.

\section{Reference}

1 Future Hospital Commission. Future hospital: caring for medical patients. A report from the Future Hospital Commission to the Royal College of Physicians. London: RCP, 2013. 